\title{
Re-entry condition for ferromagnetic superconductors
}

\author{
C. G. Kuper, M. Revzen, and A. Ron, \\ Department of Physics, Technion-Israel Institute of Technology, Haifa 32000, Israel \\ C.-R. Hu, \\ Department of Physics, Texas A \& M University, College Station, Texas 77843
}

(Received 10 June 1982; revised manuscript received 27 December 1982)

\begin{abstract}
In the phenomenological theory of re-entrant superconductors, the ratio $\zeta$ of the magnetic to superconducting free-energy densities plays a crucial role. We present arguments which suggest that $3 \leq \zeta \leq 17$ for $\mathrm{ErRh}_{4} \mathrm{~B}_{4}$, thus excluding values in excess of 100 which have appeared in the literature.
\end{abstract}

Recent phenomenological-theoretical studies ${ }^{1-4}$ have discussed various possible modes of coexistence of superconducting and magnetic order in those ternary compounds which exhibit re-entry from the superconducting state to a normal-resistive (but ferromagnetic) state.

The discussions are all based on a generalized GinzburgLandau model, first described by Krey., 6 Our notation is that of Ref. 3.

$$
F=\int d^{3} r\left[\frac{1}{2} \alpha_{s}|\psi|^{2}+\frac{1}{4} \beta_{s}|\psi|^{4}+\frac{\hbar^{2}}{2 m} \mid\left(\nabla-i \frac{2 e}{\hbar c} \overrightarrow{\mathrm{A}}|\psi|^{2}+\frac{1}{2} \alpha_{m}|M|^{2}+\frac{1}{4} \beta_{m}|M|^{4}+\frac{1}{6} \gamma_{m}|M|^{6}+\frac{1}{2} \Gamma^{2} \lambda^{2}|\nabla \overrightarrow{\mathrm{M}}|^{2}+\frac{H^{2}}{8 \pi}\right]\right.
$$

where

$$
\begin{aligned}
& \alpha_{m}=-\left|\alpha_{m 0}\right|\left(1-T / T_{m}\right) ; \\
& \alpha_{s}=-\left|\alpha_{s 0}\right|\left(1-T / T_{c 1}\right) .
\end{aligned}
$$

An important parameter in these studies is $\zeta$ :

$$
\zeta=F_{m}^{(\mathrm{GL})}(0) / F_{s}^{(\mathrm{GL})}(0),
$$

the ratio of the Ginzburg-Landau magnetic and superconducting free-energy densities, extrapolated to zero temperature. We then might ask which of the possible coexistence modes is likely to be realized. The answer depends crucially on $\zeta$. Re-entry from the helical spin-density-wave state to normal ferromagnetism can occur ${ }^{7}$ only if

$$
\left|\alpha_{m 0}\right| / 8 \pi^{1 / 2} \Gamma \leq \zeta .
$$

If, following Ref. 1 , we take $\Gamma \sim 10^{-2}$ and $\left|\alpha_{m 0}\right| \mid \simeq 15,{ }^{7}$ the criterion for re-entry is $\zeta \geq 100$. Moreover, the linear spindensity-wave state $^{2}$ is not stable relative to the vortex state if $\zeta$ becomes much smaller than 100 .

In the literature, values of $\zeta$ for $\mathrm{ErRh}_{4} \mathrm{~B}_{4}$ as high ${ }^{2}$ as 500 and as $l o w^{3}$ as 10 have been proposed. In the current Brief Report, we present a series of arguments which support values near the lower end of this range. We also discuss briefly the value of $\left|\alpha_{m 0}\right|$, since the re-entry condition (4) depends on this dimensionless number.

Values of $\left|\alpha_{m 0}\right|$ used in previous studies range from ${ }^{1,8}$ $\left|\alpha_{m 0}\right| \simeq 14$ to $\left|\alpha_{m 0}\right| \simeq 60 .^{3}$ But there is very recent direct evidence that $\left|\alpha_{m 0}\right|$ may be as low as 5. Behroozi et al. ${ }^{9}$ have measured the paramagnetic susceptibility of polycrystalline $\mathrm{ErRh}_{4} \mathrm{~B}_{4}$ in the absence of superconductivity. Upon extrapolating to zero applied field, they find $\left|\alpha_{m 0}\right|=5.5$ \pm 0.5 . Jaric ${ }^{10}$ had previously proposed a similarly "low" value, $\left|\alpha_{m 0}\right| \simeq 7.5$.

To establish reasonable bounds on $\zeta$, we shall assume $5 \leqslant\left|\alpha_{m 0}\right| \leqslant 15$. With the lowest estimate, $\left|\alpha_{m 0}\right|=5$, the re-entry condition from the helical state [Eq. (4)] becomes $\zeta \geq 35$. The estimates which we shall present here suggest that $\zeta$ cannot be nearly as high as this; we therefore believe that there are difficulties in accepting either the linear or the helical spin-density-wave states as equilibrium states of coexistence.

The parameter $\zeta$ is defined as a ratio of "magnetic" Landau parameters to "superconducting" ones. We shall first present four essentially independent estimates for the numerator of Eq. (3). Because the Landau ${ }^{6}$ theory of second-order transitions is based on a power-series expansion about the transition temperature, estimates based on thermodynamic properties near the Curie temperature should be more reliable than estimates based on properties near $T=0$.

(a) According to the Landau theory,

$$
-F_{m}(0)=\frac{1}{2} T_{m} \Delta c\left(T_{m}\right),
$$

where $\Delta c$ is the specific-heat discontinuity at the "bare" Curie temperature $T_{m}$. However, the Curie point is not accessible experimentally, because at $T_{m}$ the magnetic order is suppressed by the superconductivity. And at the re-entry point $T_{c 2}=0.9 \mathrm{~K}, \mathrm{ErRh}_{4} \mathrm{~B}_{4}$ exhibits ${ }^{11}$ a first-order transition. However, we get a naive order-of-magnitude estimate by taking $T_{m}=T_{c 2}$ and $\Delta c=c_{\max }=25 \mathrm{~J} \mathrm{~mol}^{-1} \mathrm{~K}^{-1}$ in Eq. (1), to give

$$
-F_{m}(0)=1.8 \times 10^{6} \mathrm{erg} \mathrm{cm}^{-3}
$$

How reliable is this estimate? $T_{m}$ can hardly exceed $1.3 \mathrm{~K}$, where extrapolation of the neutron-diffraction data of Sinha et al. ${ }^{12}$ gives zero magnetization. Furthermore, $c_{\max }$ is more 
likely an overestimate of $\Delta c ; \mathrm{HoRh}_{4} \mathrm{~B}_{4}$, which does have a second-order transition, has $\Delta c=14 \mathrm{~J} \mathrm{~mol}^{-1} \mathrm{~K}^{-1}$. Despite the uncertainties, Eq. (6) appears to us to be a reasonable estimate-more probably an overestimate-of $-F_{m}(0)$.

(b) We can estimate $-F_{m}(0)$ from the spontaneous magnetization $M(0)$ at $T=0$; the effective magnetic moment ${ }^{9}$ of the $\mathrm{Er}^{3+}$ ion in $\mathrm{ErRh}_{4} \mathrm{~B}_{4}$ at zero field is $\mu=5.6 \mu_{B}$, whence the spontaneous magnetization is

$$
M(0)=\mu / v=4.9 \times 10^{2} \mathrm{G} .
$$

[Here $\mu_{B}$ is the Bohr magneton, $0.9 \times 10^{-20} \mathrm{erg} \mathrm{G}^{-1}$, and $v$ is the atomic volume (i.e., the molar volume/ $N_{0}$ ) $=1.03 \times 10^{-22} \mathrm{~cm}^{3}$.] Now, from the Landau theory,

$$
M(0)=\left|\alpha_{m 0}\right| / \beta_{m},
$$

whence

$$
-F_{m}(0)=\frac{1}{4}\left|\alpha_{m 0}\right|[M(0)]^{2} .
$$

This gives bounds

$$
0.3 \times 10^{6} \mathrm{erg} \mathrm{cm}^{-3} \leq-F_{m}(0) \leq 0.9 \times 10^{6} \mathrm{erg} \mathrm{cm}^{-3} .
$$

(c) A third estimate is based on the Curie temperature $T_{m}$ :

$$
-F_{m}(0)+k T_{m} / v \text {. }
$$

With $0.7 \mathrm{~K} \leqslant T_{m} \leqslant 1.3 \mathrm{~K}$, this gives

$$
0.9 \times 10^{6} \mathrm{erg} \mathrm{cm}^{-3} \leq-F_{m}(0) \leq 1.7 \times 10^{6} \mathrm{erg} \mathrm{cm}^{-3} .
$$

We note that estimates (b) and (c) are essentially unaffected by the recent experimental suggestion ${ }^{9}$ that in $E_{r R h_{4}} B_{4}$ the coefficient $\beta_{m}$ may vanish, although in that case estimate (a) becomes meaningless.

(d) However, the same experiment which yields $\beta_{m}=0$ also gives data for $\alpha_{m}$ and $\gamma_{m}$ from which a very direct estimate may be made. Putting $\beta_{m}=0$, the minimum condition on $F$ is

$$
-F_{m}(0)=\frac{1}{3}\left|\alpha_{m 0}\right|^{3 / 2} \gamma_{m}^{-1 / 2} .
$$

Inserting the measured values ${ }^{9}\left|\alpha_{m 0}\right|=5.5, \gamma_{m}=1 \times 10^{-10}$ $\mathrm{G}^{4}$, we immediately find

$$
-F_{m}(0)=0.43 \times 10^{6} \mathrm{erg} \mathrm{cm}^{-3} .
$$

The four estimates are reasonably consistent. The most "precise" experimentally is probably (b), but it is also the most uncertain theoretically, since Eq. (8) involves the extrapolation of the Landau theory to $T=0$. Probably (d) is the most reliable. Moreover, our main interest is in finding a reliable upper bound for $\zeta$, and we therefore prefer to be "pessimistic" and to give the higher estimates of $-F_{m}$ somewhat more weight. We take as our final set of bounds on $-F_{m}(0)$

$$
0.4 \times 10^{6} \mathrm{erg} \mathrm{cm}^{-3} \leq F_{m}(0) \leq 1.5 \times 10^{6} \mathrm{erg} \mathrm{cm}^{-3} .
$$

Our next task is to estimate the GinzburgLandau-extrapolated superconducting free-energy density at zero temperature. Again we give several independent estimates.

(e) Analogously to method (a) for the magnetic free energy, we use the Ginzburg-Landau relation ${ }^{6}$

$$
-F_{s}(0)=\frac{1}{2} T_{c 1} \Delta c_{s n}\left(T_{c 1}\right)=2.7 \times 10^{5} \mathrm{erg} \mathrm{cm}^{-3} .
$$

Here - in contrast to the magnetic case $-\Delta c_{s n}$ and $T_{c 1}$ are directly measured. ${ }^{13}$ The uncertainty lies in the extrapolation of the Ginzburg-Landau theory from its region of validity near $T_{c 1}(\simeq 10 \mathrm{~K})$, down to the low-temperature region $T \leqslant 1 \mathrm{~K}$. However, we are able to correct this extrapolation. We are fortunate that the re-entry temperature is sufficiently low that all bare superconducting properties are nearly independent of the temperature. According to the BCS theory, the superconducting condensation energy is proportional to the square of the energy gap, both at $T=0$ and near $T=T_{c 1}:-F_{s} \propto \Delta^{2}$. This enables us to use the Ginzburg-Landau parameters, measured near $T_{c 1}$, to define a set of "effective" parameters valid at low temperatures. Near $T_{c 1}$, the microscopic theory gives

$$
\begin{aligned}
& \Delta / k T_{c 1}=\Theta\left(1-T / T_{c 1}\right)^{1 / 2}, \\
& \Theta=\pi[8 / 7 \zeta(3)]^{1 / 2}=3.06,
\end{aligned}
$$

while at $T=0$

$$
\Delta(0)=1.76 k T_{c 1} \text {. }
$$

Hence Eq. (15) has overestimated $-F_{s}(0)$ by a factor $(3.06 / 1.76)^{2}=3.0$; the corrected value is

$$
-F_{s}(0) \simeq 0.9 \times 10^{5} \mathrm{erg} \mathrm{cm}^{-3} .
$$

(f) A second estimate follows from the thermodynamical critical field at zero temperature:

$$
-F_{s}(0)=H_{c}^{2}(0) / 8 \pi \text {. }
$$

However, this estimate is necessarily indirect since (i) the material is of type II (except perhaps close to re-entry), and it is therefore not $H_{c}$ but rather $H_{c 1}$ and $H_{c 2}$ which are directly measured, and (ii) the material is not superconducting at $T=0$. What we can try to do is to make a BCS extrapolation of $H_{c 1}$ and $H_{c 2}$ from near $T_{c 1}$ to low temperatures. This extrapolation is reasonably consistent with the estimated value of $H_{c}$ for the non-re-entrant material ${ }^{11}$ $\mathrm{LuRh}_{4} \mathrm{~B}_{4}, H_{c}(0)=1.8 \times 10^{3} \mathrm{G}$. This yields

$$
-F_{s}(0) \simeq 1.3 \times 10^{5} \mathrm{erg} \mathrm{cm}^{-3} .
$$

(g) Our final estimate is a rather crude theoretical one:

$$
-F_{s}(0)=\frac{1}{2} N_{s}\left(k T_{c 1}\right)^{2} / \epsilon_{F},
$$

where we take $N_{s} \approx 2 \times 10^{23} \mathrm{~cm}^{-3}$ (i.e., 20 electrons per unit cell, of volume $v$ ), $T_{c 1} \simeq 10 \mathrm{~K}$, and for the Fermi energy $\epsilon_{F}$ we take $\epsilon_{F} / k=10^{4} \mathrm{~K}$. (Simple metals, e.g. $\mathrm{Na}$, tend to have $\epsilon_{F} / k=10^{5} \mathrm{~K}$, but although the electron density in $E r R h_{4} B_{4}$ is not very different from that in a simple metal, the conduction electrons are largely $d$ electrons of the $\mathrm{Rh}$, and they have a rather large effective mass). Inserting these values in $(21)$, we find

$$
-F_{s}(0) \simeq 1.3 \times 10^{5} \mathrm{erg} \mathrm{cm}^{-3} .
$$

The three estimates (e), (f), and (g) are consistent. We therefore believe that

$$
0.9 \times 10^{5} \mathrm{erg} \mathrm{cm}^{-3} \leq-F_{s}(0) \leq 1.3 \times 10^{5} \mathrm{erg} \mathrm{cm}^{-3}
$$

represent reasonable bounds, and especially that the lower bound is reliable.

Finally, from Eqs. (14) and $(23), \zeta \equiv F_{m}(0) / F_{s}(0)$ has as 
its bounds

$$
3 \leq \zeta \leq 17
$$

Thus, while the estimate $\zeta=10$ of Ref. 3 may conceivably be slightly on the low side, the values necessary to give reentry from the helical state ${ }^{1}(\zeta \geq 100$, or even $\geq 35$ ) would appear to be very implausible. ${ }^{14}$

Neutron-diffraction experiments ${ }^{12}$ on $\mathrm{ErRh}_{4} \mathrm{~B}_{4}$ appear to favor some type of a linearly polarized spin-density-wave state. Should it be confirmed that this observed state is simply the plane-wave-like linear spin-density-wave state envisioned in Ref. 2, our low value for $\zeta$ would lead to the conclusion that the simple Ginzburg-Landau approach is inadequate.

\section{ACKNOWLEDGMENTS}

Work performed at Technion-Israel Institute of Technology was supported in part by grants from Kernforschungszentrum Karlsruhe and the Technion's Research Fund. Work performed at Texas A\&M University was supported by National Science Foundation Grant No. DMR82-05697.
${ }^{1}$ E. I. Blount and C. M. Varma, Phys. Rev. Lett. 42, 1079 (1979).

${ }^{2}$ H. S. Greenside, E. I. Blount, and C. M. Varma, Phys. Rev. Lett. 46, 49 (1981).

${ }^{3}$ C. G. Kuper, M. Revzen, and A. Ron, Phys. Rev. Lett. 44, 1545 (1980); Solid State Commun. 36, 533 (1980).

${ }^{4}$ M. Tachiki, H. Matsumoto, T. Koyama, and H. Umezawa, Solid State Commun. 34, 19 (1980), and references contained therein; C.-R. Hu and T.-E. Ham, Physica B 108, 1041 (1981).

${ }^{5}$ U. Krey, Int. J. Magn. $\underline{3}, 65$ (1972); $\underline{4}, 153$ (1973). For simplicity, we neglect anisotropy. Actually $\mathrm{ErRh}_{4} \mathrm{~B}_{4}$ forms tetragonal crystals with a magnetically very hard $c$ axis [see F. Behroozi, G. W. Crabtree, S. A. Campbell, M. Levy, D. R. Snider, D. C. Johnston, and B. T. Matthias, Solid State Commun. 39, 1041 (1981); Ternary Superconductors, edited by G. K. Shenoy, B. D. Dunlap, and F. Y. Fradin (North-Holland, Amsterdam, 1981), p. 155; G. W. Crabtree, F. Behroozi, S. A. Campbell, and D. G. Hinks, report (unpublished).

${ }^{6}$ L. D. Landau, E. M. Lifshitz, and L. P. Pitaevski, Statistical Physics, 3rd ed. (Pergamon, Oxford, 1980), Part I, Chap. 14; Part II, Chap. 5.

${ }^{7}$ The free energy of the helically magnetized phase can be shown to be

$$
-F_{\text {hel }}(T)=-F_{s}(T)-F_{m}(T)\left(1-8 \pi^{1 / 2} \Gamma /\left|\alpha_{m 0}\right|\right) .
$$

The re-entry temperature is fixed by the condition
$F_{\text {hel }}(T)=F_{m}(T)$. This equation has no solution unless condition (4) is satisfied.

${ }^{8}$ Blount and Varma (Ref. 1) assume $\left|\alpha_{0}^{\prime}\right| \simeq \mid$ where $\alpha^{\prime}=\alpha-4 \pi$; i.e., $\left|\alpha_{m 0}\right| \approx 13$ or 14 .

${ }^{9}$ Behroozi, Crabtree, Campbell, Levy, Snider, Johnston, and Matthias, Ref. 5; Crabtree, Behroozi, Campbell, and Hinks, Ref. 5.

${ }^{10}$ M. V. Jarić, Phys. Rev. B 22, 3503 (1979), Ref. 22.

${ }^{11}$ H. B. MacKay, L. D. Woolf, M. B. Maple, and D. C. Johnston, Phys. Rev. Lett. 42, 918 (1979).

${ }^{12}$ S. K. Sinha, G. W. Crabtree, D. G. Hinks, and H. Mook, Phys. Rev. Lett. 48, 950 (1982).

${ }^{13}$ W. A. Fertig, D. C. Johnston, L. E. DeLong, R. W. McCallum, M. B. Maple, and B. T. Matthias, Phys. Rev. Lett. 38, 987 (1977); D. E. Moncton, D. B. McWhan, J. Eckert, G. Shirane, and W. Tomlinson, ibid. 39,1164 (1977).

${ }^{14}$ This conclusion becomes even less doubtful if one realizes that the lower critical $\zeta$ must still be compared with the lower estimated values of $\zeta$ due to their common dependences on the value of $\left|\alpha_{m 0}\right|$. Also, in the model in which $\beta_{m}=0, \gamma_{m} \neq 0$, the factor 8 in Eq. (4) must still be changed to 6 , changing the condition $\zeta \geq 35$ to $\zeta \geq 47$. Finally, one must still realize that the observed re-entry of the coexistence state occurred near $T_{c 2}$ rather than near $T=0$, which requires an even larger $\zeta$. 